\title{
Nationwide outcomes of surgical embolectomy for acute pulmonary embolism
}

\author{
Arman Kilic, MD, ${ }^{\mathrm{a}}$ Ashish S. Shah, MD, ${ }^{\mathrm{a}}$ John V. Conte, MD, ${ }^{\mathrm{a}}$ and David D. Yuh, MD
}

Objective: The aim of the present study was to review nationwide outcomes of surgical embolectomy for acute pulmonary embolism.

\begin{abstract}
Methods: Adult patients undergoing surgical embolectomy for acute pulmonary embolism from 1999 to 2008 were identified in the weighted Nationwide Inpatient Sample. The primary endpoint was inpatient mortality. Multivariate logistic regression analysis incorporating significant univariate predictors $(P<.2)$ was conducted to identify independent predictors of inpatient mortality.
\end{abstract}

Results: There were 2709 eligible patients identified as undergoing surgical embolectomy for acute pulmonary embolism during the study period. The mean age was $57.0 \pm 16.0$ years. Of the patients, $1242(45.8 \%)$ were women. A total of 280 patients $(10.3 \%)$ had undergone thrombolysis before surgical embolectomy. The overall inpatient mortality rate was $27.2 \%$. On multivariate analysis, an increasing Charlson comorbidity index (odds ratio, $1.37 ; 95 \%$ confidence interval, $1.12-1.69 ; P=.003$ ) significantly increased the odds of inpatient mortality. In addition, blacks were more than twofold more likely to die during hospitalization than whites (odds ratio, $2.29 ; 95 \%$ confidence interval, $1.18-4.46 ; P=.02$ ). Although age, payment type, hospital location (urban versus rural), hospital embolectomy volume, and surgeon embolectomy volume were associated with inpatient mortality on univariate analysis (each $P<.2$ ), none of these factors correlated with mortality in the multivariate model.

Conclusions: This large-cohort analysis of more than 2700 patients demonstrates a nationwide inpatient mortality rate of $27.2 \%$ after pulmonary embolectomy. Although patient factors affect mortality, the arena of care appears to have no significant effect on operative outcomes. This suggests that it might be more prudent for centers with qualified surgeons to avoid delays in treatment, rather than transfer care because of a perception of improved outcomes. (J Thorac Cardiovasc Surg 2013;145:373-7)

Approximately 300,000 people die of acute pulmonary embolism (PE) in the United States annually. ${ }^{1,2}$ In the setting of a massive PE, the infusion of heparin or a thrombolytic agent such as alteplase is generally recommended, although bleeding complications remain a real concern with the latter. ${ }^{3,4}$ Surgical embolectomy is a therapeutic option for patients who have contraindications to thrombolysis or who have persistent hypotension after initial therapy. Pulmonary embolectomy has also been associated with favorable results in patients with preserved hemodynamics but with evidence of moderate to severe right ventricular dysfunction on echocardiography. ${ }^{5}$ Regardless of the indication, previous outcome analyses for pulmonary embolectomy have generally been limited by

\footnotetext{
From the Division of Cardiac Surgery, ${ }^{\mathrm{a}}$ Department of Surgery, Johns Hopkins Medical Institutions, Baltimore, Md; and Section of Cardiac Surgery, ${ }^{\mathrm{b}}$ Department of Surgery, Yale University School of Medicine, New Haven, Conn.

This study was supported by departmental funds from the Department of Surgery, Johns Hopkins Hospital, Baltimore, MD.

Disclosures: Authors have nothing to disclose with regard to commercial support.

Received for publication Nov 11, 2011; revisions received Jan 9, 2012; accepted for publication Jan 24, 2012; available ahead of print Feb 20, 2012.

Address for reprints: David D. Yuh, MD, Section of Cardiac Surgery, Department of Surgery, Yale University School of Medicine, 330 Cedar Street, Boardman 204,

New Haven, CT 06510 (E-mail: david.yuh@yale.edu).

$0022-5223 / \$ 36.00$

Copyright (c) 2013 by The American Association for Thoracic Surgery

doi:10.1016/j.jtcvs.2012.01.066
}

small patient cohorts. In the present study, we evaluated the nationwide outcomes of surgical embolectomy for acute PE.

\section{METHODS \\ Data Source}

The Nationwide Inpatient Sample served as the data source for the present analysis. This database is 1 of several registries initiated as a part of the Healthcare Cost and Utilization Project, developed by the Agency for Healthcare Research and Quality. The Nationwide Inpatient Sample contains data from 1050 participating institutions in 44 states, representing a $20 \%$ stratified sample of community hospitals. ${ }^{6}$ By using a weighting strategy, these data reflect approximately $95 \%$ of all hospital discharges within the United States. The database does not contain any patient or center identifiers. Our institutional review board approved the study protocol

\section{Study Cohort}

The study population in the present analysis consisted of adult (age $\geq 18$ years) patients undergoing surgical embolectomy for acute PE. The International Classification of Diseases, Ninth Revision (ICD-9) codes were used to identify this patient subset. Moreover, patients with acute PE were initially identified using ICD-9 codes 415.1, 415.11, 415.12, and 415.19. Patients with PE complicating pregnancy or childbirth were excluded from the analysis (codes 634-638 with .6, 639.6, or 673.0-673.8) Pulmonary surgical embolectomy was identified by the ICD-9 code 38.05. The use of thrombolytics was identified by code 99.10

\section{Statistical Analysis}

The primary outcome of interest was inpatient mortality, defined as death occurring during the admission in which surgical embolectomy 


\section{Abbreviations and Acronyms \\ ICD-9 = International Classification of Diseases, Ninth Revision \\ $\mathrm{PE} \quad=$ pulmonary embolism}

\section{Logistic Regression Analysis}

Seven covariates were found to be associated with inpatient mortality after pulmonary embolectomy in our univariate logistic regression analysis (each $P<.2$ ). Those covariates included age, race, Charlson comorbidity index, payment type, hospital location, hospital embolectomy volume, and surgeon embolectomy volume. On multivariate logistic regression analysis incorporating these 7 variables, only 2 variables persisted as significant risk factors for inpatient mortality: increasing comorbidity index and black race. Moreover, for each 1-point increase in the comorbidity index, there was a $37 \%$ increase in inpatient mortality $(P=.003$; Table 3$)$. With respect to race, blacks were $129 \%$ more likely to die after surgical embolectomy than whites $(P=.02)$.

None of the hospital characteristics was found to independently predict operative mortality on multivariate analysis. In limiting the analysis to urban centers, in which the vast majority of patients underwent embolectomy (97.1\%), the operative mortality rates were comparable after stratifying patients according to teaching status and size of the institution (Figure 1). Furthermore, to ensure that delays in treatment that could possibly occur with the transfer of patients were not significantly accounting for the comparable outcomes between hospitals, a secondary analysis was performed that excluded transferred patients. The operative mortality in this subanalysis remained comparable among the various types of hospitals stratified according to teaching status, size, and location (each $P>.05$ ).

\section{DISCUSSION}

Selecting the initial therapeutic strategy for PE generally depends on assessing the mortality risk from the embolic load and the risk of bleeding from thrombolytic agents. Heparin is recommended for patients who are hemodynamically stable and have no evidence of right ventricular dysfunction on echocardiography, because they represent the cohort with the lowest PE-related mortality risk. In patients with submassive PE, defined by hemodynamic stability but echocardiographic evidence of right ventricular dysfunction, the utility of thrombolysis is controversial, with a randomized study failing to demonstrate a significant reduction in mortality compared with heparin alone. ${ }^{7}$ Favorable results with surgical embolectomy in submassive PE have been reported. ${ }^{8,9}$ In the case of massive PE with hemodynamic instability, a meta-analysis of several randomized trials demonstrated a significant reduction in recurrent PE or death with thrombolysis compared with heparin, but an international multicenter registry found no such reduction. ${ }^{3,10}$ In addition to its increasing use in submassive PE, surgical embolectomy is generally recommended in cases of persistent hemodynamic instability after initial systemic thrombolysis, in cases of massive PE in which thrombolysis is contraindicated 
TABLE 1. Baseline patient and clinical differences stratified by survival to discharge

\begin{tabular}{|c|c|c|c|c|}
\hline Variable & All patients $(n=2709)$ & Survivors $(n=1972)$ & Operative mortality $(n=737)$ & $P$ value* \\
\hline Age $(y)$ & $57.0 \pm 16.0$ & $55.9 \pm 15.8$ & $60.0 \pm 16.1$ & .01 \\
\hline Women & $1242 / 2709(45.8)$ & $880 / 1972(44.6)$ & $362 / 737(49.1)$ & .42 \\
\hline Race & & & & .17 \\
\hline White & $1553 / 2099(74.0)$ & 1209/1572 (76.9) & $344 / 527(65.3)$ & \\
\hline Black & $347 / 2099(16.5)$ & $229 / 1572(14.6)$ & $118 / 527(22.4)$ & \\
\hline Hispanic & $84 / 2099(4.0)$ & $61 / 1572(3.9)$ & 23/527 (4.4) & \\
\hline Other & $115 / 2099(5.5)$ & $73 / 1572(4.6)$ & $42 / 527(8.0)$ & \\
\hline Charlson comorbidity index & $1.9 \pm 1.1$ & $1.8 \pm 1.0$ & $2.2 \pm 1.2$ & .002 \\
\hline Payment status & & & & .17 \\
\hline Self-pay or privately insured & $1333 / 2709(49.2)$ & 1017/1972 (51.6) & $316 / 737(42.9)$ & \\
\hline Medicare & $1057 / 2709(39.0)$ & $712 / 1972(36.1)$ & $345 / 737(46.8)$ & \\
\hline Medicaid & $211 / 2709(7.8)$ & $165 / 1972(8.4)$ & $46 / 737(6.2)$ & \\
\hline Other & $108 / 2709(4.0)$ & $78 / 1972(4.0)$ & $30 / 737(4.1)$ & \\
\hline Thrombolysis & $280 / 2709(10.3)$ & 196/1972 (9.9) & 84/737 (11.4) & .65 \\
\hline
\end{tabular}

Continuous data are presented as mean \pm standard deviation and frequency data as $\mathrm{n}(\%)$. $P$ value for comparison between survivors to discharge and patients with operative mortality.

owing to a high bleeding risk, and in cases in which there is not sufficient time to infuse thrombolytic agents. ${ }^{11}$

\section{Study Rationale}

Most of the previous data describing the outcomes of surgical embolectomy in the setting of acute PE have been handicapped by small patient cohorts, ranging from 7 to 47 subjects in the modern era. ${ }^{5,9,12-15}$ In our study, we reviewed the nationwide outcomes of surgical embolectomy for acute PE in a large cohort of more than 2700 patients. Particular attention was paid to the patient and hospital factors that predicted inpatient mortality.

\section{Inpatient Mortality}

A principal finding in our analysis was that surgical embolectomy was associated with a high inpatient mortality of $27.2 \%$. This was not surprising, given that acute PE, in and of itself, is known to be associated with significant mortality risk, especially when prompt diagnosis and treatment are not initiated. The International Cooperative Pulmonary Embolism Registry studied more than 2450 patients from multiple centers and found an all-cause 14-day and 90-day mortality rate of $11.4 \%$ and $17.4 \%$, respectively, in all patients with PE. ${ }^{10}$ The mortality rates are particularly high in cases of PE with associated cardiac arrest, cardiogenic shock, or hypotension, at about $60 \%, 23 \%$, and $14 \%$, respectively. ${ }^{16}$

With regard to the outcomes after embolectomy, there have been several reports of institutional experiences in the modern era. A study of 47 patients reported a low operative mortality of $6 \% .^{9}$ Importantly, however, a significant proportion of patients in their analysis were hemodynamically stable, with right ventricular dysfunction as the indication for surgery. Similarly, an analysis of 29 patients demonstrated a 30-day mortality of $11 \%$, again with some patients being hemodynamically stable, with right ventricular dysfunction as the surgical indication. ${ }^{5}$ These reports have raised the notion that an aggressive approach with early surgical intervention before hemodynamic instability ensues might be associated with favorable outcomes, although another recent study of 25 critically ill patients receiving inotropic support and undergoing embolectomy for acute PE also demonstrated excellent outcomes, with an operative mortality of $8 \% .^{12}$

Although the optimal timing for surgical intervention remains to be determined, it does appear that operative mortality rates, in general, have improved over time. ${ }^{17}$ In a recent review, the cumulative operative mortality for

TABLE 2. Baseline hospital differences stratified by survival to discharge

\begin{tabular}{|c|c|c|c|c|}
\hline Variable & All patients $(n=2709)$ & Survivors $(n=1972)$ & Operative mortality $(n=737)$ & $P$ value* \\
\hline Teaching hospital & $1992 / 2709(73.5)$ & 1468/1972 (74.4) & $523 / 737(71.0)$ & .43 \\
\hline Urban location & $2630 / 2709(97.1)$ & 1932/1972 (98.0) & 698/737 (94.7) & .08 \\
\hline Hospital size & & & & .42 \\
\hline Small & $77 / 2709(2.8)$ & $59 / 1972(3.0)$ & $18 / 737(2.4)$ & \\
\hline Medium & $449 / 2709(16.6)$ & $347 / 1972(17.6)$ & $102 / 737(13.8)$ & \\
\hline Large & $2183 / 2709(80.6)$ & $1566 / 1972(79.4)$ & $617 / 737(83.7)$ & \\
\hline Hospital annual volume & $2.3 \pm 1.6$ & $2.4 \pm 1.7$ & $2.1 \pm 1.3$ & .04 \\
\hline Surgeon annual volume & $0.7 \pm 0.7$ & $0.8 \pm 0.7$ & $0.6 \pm 0.6$ & .02 \\
\hline
\end{tabular}

Continuous data are presented as mean \pm standard deviation and frequency data as $\mathrm{n}(\%) . * P$ value for comparison between survivors to discharge and patients with operative mortality. 
TABLE 3. Multivariate logistic regression analysis* for inpatient mortality after pulmonary embolectomy

\begin{tabular}{lcc}
\hline \multicolumn{1}{c}{ Covariate } & OR $(\mathbf{9 5} \% \mathbf{C I})$ & $\boldsymbol{P}$ value \\
\hline Age (increasing) & $1.02(1.00-1.03)$ & .11 \\
Charlson Comorbidity Index (increasing) & $1.37(1.12-1.69)$ & .003 \\
Race & & \\
$\quad$ White & Reference & \\
Black & $2.29(1.18-4.46)$ & .02 \\
Hispanic & $1.60(0.40-6.41)$ & .50 \\
Other & $2.15(0.85-5.41)$ & .10 \\
Payment type & & \\
$\quad$ Private or self-pay & Reference & \\
$\quad$ Medicare & $1.90(0.78-4.64)$ & .16 \\
Medicaid & $2.18(0.70-6.83)$ & .18 \\
Other & $2.27(0.61-8.49)$ & .22 \\
Rural hospital & $2.65(0.62-11.3)$ & .19 \\
Hospital annual volume (decreasing) & $1.06(0.90-1.24)$ & .47 \\
Surgeon annual volume (decreasing) & $1.24(0.83-1.86)$ & .29 \\
\hline
\end{tabular}

$O R$, Odds ratio; $C I$, confidence interval. *Variables included in multivariate model were associated $(P<.2)$ with inpatient mortality on univariate logistic regression analysis.

surgical embolectomy was found to have significantly improved from $30 \%$ to $35 \%$ before 1999 to $19 \%$ thereafter. $^{18}$ Whether this reduction in mortality rates has resulted from earlier diagnosis, better surgical techniques, improved perioperative care, and/or changes in the process of care that might reduce the interval to treatment remains unknown.

\section{Predictors of Mortality}

Several studies have suggested that the severity of hemodynamic instability correlates with mortality after surgical embolectomy. More specifically, patients experiencing cardiac arrest and requiring cardiopulmonary resuscitation before surgical embolectomy have been demonstrated to be at greater risk of postoperative mortality in these studies. ${ }^{13,19}$ This notion has been contradicted, however, by more recent reports of low operative mortality even in patients with preoperative cardiac arrest. ${ }^{12}$ In our study, we did not include cardiopulmonary resuscitation in the multivariate model, because we could not determine whether cardiac

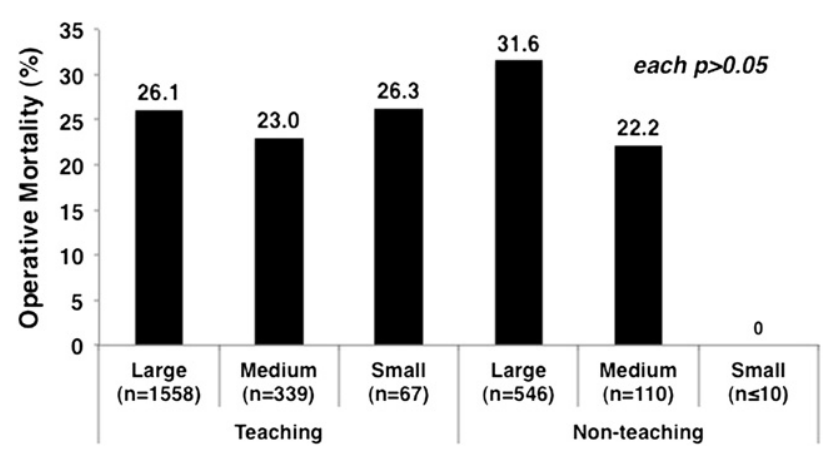

FIGURE 1. Operative mortality in urban hospitals, stratified by teaching status and hospital size. arrest occurred pre- or postoperatively in the Nationwide Inpatient Sample.

In our analysis, increasing comorbidity index and black race were independent predictors of operative mortality. Although the finding that a greater comorbidity burden correlated with increased mortality was not unexpected, we were surprised to find that racial disparities in outcomes persisted after adjusting for other patient and hospital factors. It is unclear from our analysis whether this observed effect of race on inpatient mortality after embolectomy relates to biologic or socioeconomic factors, or both. A previous analysis of more than 15,000 patients inclusive of all patients with $\mathrm{PE}$, regardless of therapeutic modality, similarly found that blacks had significantly greater odds of 30-day mortality relative to white. ${ }^{20}$ This finding was also similar to our analysis in that this effect was independent of other patient or clinical factors such as insurance status and hospital volume.

\section{Hospital Factors}

Surgical embolectomy is generally not a complex, technically challenging operation. This could at least partially explain why surgeon procedural volume did not affect the operative outcomes in our analysis. However, one could postulate that the hospital procedural volume should affect the operative outcomes because institutions with more experience in performing embolectomy might have standardized clinical pathways in place and might have better optimized processes of care that lead to earlier diagnosis and treatment. This, however, was not found in our study, perhaps because the annual hospital volumes were so low, with only 2.3 cases performed annually on average.

In addition to procedural volume, none of the other hospital or system factors were found to correlate with operative mortality. These findings persisted after excluding patients who were transferred from other hospitals. Therefore, we believe these data collectively suggest that the arena of care has no significant effect on outcomes after pulmonary embolectomy and that patient factors are more important. As such, from our analysis, it appears that transferring patients because of a perception of improved outcomes should be avoided, provided a qualified surgeon is available to perform the embolectomy, especially given the risk of clinical deterioration during the delay in treatment that would accompany such transfers.

\section{Study Limitations}

A major study limitation was that we were unable to include certain variables that might have affected operative mortality, such as hemodynamic instability or cardiac arrest occurring before surgical intervention. Although ICD-9 codes are available for these, it is unclear from the Nationwide Inpatient Sample in which sequence these adverse events occurred in relation to the surgical embolectomy. Other 
variables such as the underlying cause of PE or the interval to initial treatment also were not included. We also did not examine the use of catheter-based embolectomy in the present analysis. In terms of procedural volume, we only evaluated pulmonary embolectomy volumes, although one could postulate that the overall cardiac volume or volume of cardiopulmonary bypass cases could have an effect on the outcomes of embolectomy. Finally, there are inherent limitations to large administrative data sets, including the Nationwide Inpatient Sample, that we could not eliminate, including errors in data entry and missing data.

\section{CONCLUSIONS}

We analyzed more than 2700 cases of surgical embolectomy for acute PE in this nationwide cohort. Overall inpatient mortality was $27.2 \%$. Significant predictors of inpatient mortality after embolectomy were limited to patient factors and included increasing comorbidity burden and race. Additional research to delineate the causes for these racial disparities in outcomes is warranted. Furthermore, hospital factors such as procedural volume, teaching status, or hospital size had no independent effect on the outcomes. These data collectively suggest that it might be more prudent for centers with qualified surgeons to avoid delays in treatment rather than transfer care on the basis of a perception of improved outcomes. This is especially important when considering that such delays that often accompany a transfer of care can lead to clinical deterioration of the patient and exponential increases in mortality risk.

\section{References}

1. Silverstein MD, Heit JA, Mohr DN, Petterson TM, O'Fallon WM, Melton LJ III. Trends in the incidence of deep vein thrombosis and pulmonary embolism: a 25year population-based study. Arch Intern Med. 1998;158:585-93.

2. Heit JA, Cohen AT, Anderson FA Jr. Estimated annual number of incident and recurrent, non-fatal and fatal venous thromboembolism (VTE) events in the US. Blood. 2005;106:267a.

3. Wan S, Quinlan DJ, Agnelli G, Eikelboom JW. Thrombolysis compared with heparin for the initial treatment of pulmonary embolism: a meta-analysis of the randomized controlled trials. Circulation. 2004;110:744-79.
4. Buller HR, Agnelli G, Hull RD, Hyers TM, Prins MH, Raskob GE. Antithrombotic therapy for venous thromboembolic disease: the Seventh ACCP Conference on Antithrombotic and Thrombolytic Therapy. Chest. 2004;126:401S-28S.

5. Aklog L, Williams CS, Byrne JG, Goldhaber SZ. Acute pulmonary embolism: a contemporary approach. Circulation. 2002;105:1416-9.

6. Overview of the Nationwide Inpatient Sample. Available at: http://www.hcup-us. ahrq.gov/nisoverview.jsp. Accessed November 8, 2011.

7. Konstantinides S, Geibel A, Heusel G, Heinrich F, Kasper W. Heparin plus alteplase compared with heparin alone in patients with submassive pulmonary embolism. N Engl J Med. 2002;347:1143-50

8. Ahmed P, Khan AA, Smith A, Pagala M, Abrol S, Cunningham JN Jr, et al. Expedient pulmonary embolectomy for acute pulmonary embolism: improved outcomes. Interact Cardiovasc Thorac Surg. 2008;7:591-4.

9. Leacche M, Unic D, Goldhaber SZ, Rawn JD, Aranki SF, Couper GS, et al. Modern surgical treatment of massive pulmonary embolism: results in 47 consecutive patients after rapid diagnosis and aggressive surgical approach. J Thorac Cardiovasc Surg. 2005;129:1018-23.

10. Goldhaber SZ, Visani L, De Rosa M. Acute pulmonary embolism: clinical outcomes in the International Cooperative Pulmonary Embolism Registry (ICOPER). Lancet. 1999;353:1386-9.

11. Kearon C, Kahn SR, Agnelli G, Goldhaber S, Raskob GE, Comerota AJ. Antithrombotic therapy for venous thromboembolic disease: American College of Chest Physicians Evidence-Based Clinical Practice Guidelines. Chest. 2008; 133:454S-545S.

12. Kadner A, Schmidli J, Schonhoff F, Krahenbuhl E, Immer F, Carrel T, et al. Excellent outcome after surgical treatment of massive pulmonary embolism in critically ill patients. J Thorac Cardiovasc Surg. 2008;136:448-51.

13. Dauphine C, Omari B. Pulmonary embolectomy for acute massive pulmonary embolism. Ann Thorac Surg. 2005;79:1240-4.

14. Konstantinov IE, Saxena P, Koniuszko MD, Alvarez J, Newman MA. Acute massive pulmonary embolism with cardiopulmonary resuscitation: management and results. Tex Heart Inst J. 2007;34:41-5.

15. Greelish JP, Leacche M, Solenkova NS, Ahmad RM, Byrne JG. Improved midterm outcomes for type A (central) pulmonary emboli treated surgically. J Thorac Cardiovasc Surg. 2011;142:1423-9.

16. Kasper W, Konstantinides S, Geibel A, Olschewski M, Heinrich F, Grosser KD et al. Management strategies and determinants of outcome in acute major pulmonary embolism: results of a multicenter registry. J Am Coll Cardiol. 1997;30: 1165-71.

17. Meyer G, Tamisier D, Sors H, Stern M, Vouhe P, Makowski S, et al. Pulmonary embolectomy: a 20-year experience at one center. Ann Thorac Surg. 1991;51: 232-6.

18. Samoukovic G, Malas T, deVarennes B. The role of pulmonary embolectomy in the treatment of acute pulmonary embolism: a literature review from 1968 to 2008. Interact Cardiovasc Thorac Surg. 2010;11:265-70.

19. Schmid C, Zietlow S, Wagner TO, Laas J, Borst HG. Fulminant pulmonary embolism: symptoms, diagnostics, operative technique, and results. Ann Thorac Surg. 1991;52:1102-5.

20. Ibrahim SA, Stone RA, Obrosky DS, Sartorius J, Fine MJ, Aujesky D. Racial differences in 30-day mortality for pulmonary embolism. Am J Public Health. 2006 96:2161-4. 